\title{
Norma de Interoperabilidad de los sistemas de telecontrol y su aplicación práctica en el Centro Nacional de Tecnología de Re- gadíos (CENTER)
}

Cervantes Díaz-Toledo, Alfonso ${ }^{1}$, Madurga del Cura, Cristina², Muñoz Sopeña, Daniel², Rodríguez Fernández, Diego $^{2}$, Sánchez de Ribera González, Alejandro², Zazo Salinero, Teresa², Nicolae Tudorache, Adrian³ ${ }^{3}$ González Vicente, David $^{3}$

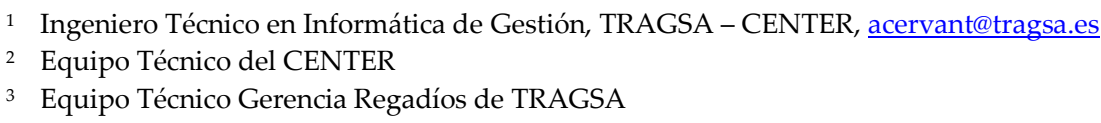

Resumen: El pasado mes de junio se publicó la norma UNE-318002-3 “Técnicas de riego. Telecontrol de zonas regables. Parte 3: Interoperabilidad", en cuya elaboración el CENTER ha participado de forma muy activa desde que en el año 2004 se comenzara a trabajar en este tema.

La interoperabilidad entre los diferentes productos destinados a la monitorización, control y gestión del regadío modernizado es un objetivo de creciente importancia debido a la heterogeneidad entre las soluciones que el mercado ofrece, sobre todo en lo referente a tecnologías y funcionalidades.

Por ello, esta norma proporciona los requisitos mínimos de estructura de datos para la transmisión de los mismos entre los diferentes equipos, dispositivos y sensores que se puedan instalar en una red de riego, aunque estos sean de diferentes empresas o utilicen softwares distintos, haciendo realidad una agricultura digital.

Parte del trabajo que conlleva la publicación de una norma es comprobar la robustez y fiabilidad de los contenidos de la misma por lo que se consideró necesario el despliegue de diferentes soluciones de mercado en una instalación de referencia. El objetivo era realizar una nueva fase de pruebas y ensayos en una instalación real al completo. Dicha instalación de referencia es la finca experimental del Centro Nacional de Tecnología de Regadíos (CENTER).

Para realizar las pruebas previstas, se han sustituido parte de las unidades remotas existentes por equipos de otros sistemas interoperables, destacando las siguientes características:

- emplean diferentes tecnologías de comunicaciones, como el GPRS y radiofrecuencia de banda libre, responden a diferentes arquitecturas, con topologías en árbol (enlaces mediante concentradoras) y en estrella (conexión directa con el software de control);

- su software de control puede estar instalado localmente o en la nube del proveedor del sistema;

El objetivo de este trabajo es mostrar la experiencia del CENTER al implantar un sistema de telecontrol interoperable y los resultados de las pruebas realizadas a lo largo de 3 campañas de riego.

Palabras clave: telecontrol, interoperabilidad, ensayos, CENTER. 


\title{
Interoperability standard for remote systems and its practical application in the National Center for Irrigation Technology (CENTER).
}

Cervantes Díaz-Toledo, Alfonso ${ }^{1}$, Madurga del Cura, Cristina ${ }^{2}$, Muñoz Sopeña, Daniel ${ }^{2}$, Rodríguez Fernández, Diego $^{2}$, Sánchez de Ribera González, Alejandro² ${ }^{2}$ Zazo Salinero, Teresa² ${ }^{2}$ Nicolae Tudorache, Adrian³ , González Vicente, David ${ }^{3}$

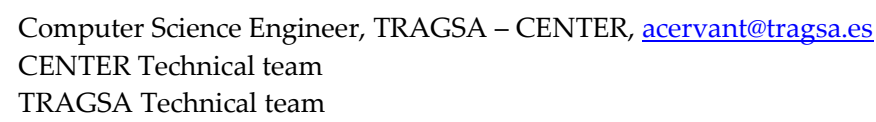

\begin{abstract}
The interoperability between the different products for the monitoring, control and management of modernized irrigation is an objective of increasing importance due to the heterogeneity between solutions that the market offers, especially with regard to technologies and functionalities.

Therefore, the standard UNE-318002-3 “Técnicas de riego. Telecontrol de zonas regables. Parte 3: Interoperabilidad" provides the minimum information structure requirements for the transmission of data between the different equipment, devices and sensors that can be installed in an irrigation network, even if they are from different companies or use different software, making it a reality a digital agriculture.

Part of the work involved in the publication of a standard is to verify the robustness and reliability of its contents, which is why it was considered necessary to deploy different market solutions in a reference facility. The objective was to carry out a new phase of test and trials in a real installation in full. This reference facility is the National Center for Irrigation Technology (CENTER).
\end{abstract}

Keywords: interoperability, remote systems, CENTER 


\section{Introducción}

Los fabricantes de sistemas de telecontrol de regadíos ofrecen soluciones diversas, sobre todo hablando de tecnologías empleadas. Dichas soluciones son cerradas para el ususario, haciéndole esclavo de su sistema.

De acuerdo con la definición de Instituto Europeo de Estándares de Telecomunicaciones (ETSI) la interoperabilidad es la capacidad de dos o más sistemas diferentes o componentes para intercambiar datos y utilizar la información intercambiada

El proyecto de interoperabilidad comenzó en el año 2008 a nivel nacional y europeo. Sin embargo, enseguida pasó a ser un proyecto internacional debido a la gran preocupación de todos los países en este tema, trabajando de forma paralela tanto en el CTN318 "Riegos" como en el ISO/TC23/SC18 "Irrigation and drainage equipment and systems". En ambos comités participan fabricantes, usuarios y diversas instituciones, públicas y privadas, que aportan sus conocimientos y permiten desarrollar una norma objetiva y útil para el sector.

En la primera fase del proyecto se definió, teóricamente, cómo abordar la interoperabilidad en el regadío. Más concretamente, en los sistemas de telecontrol, de forma que en una misma instalación pudieran convivir diferentes dispositivos.

Tras el desarrollo teórico y las comprobaciones en laboratorio y bancos de ensayo controlados, se decidió instalar en el Centro Nacional de Tecnología de Regadíos (CENTER) distintos sistemas de telecontrol interoperables y una única herramienta de gestión, con el objetivo de verificar lo indicado en la futura norma en una instalación real. Estos trabajos se han llevado a cabo durante 3 campañas de riego, desde el año 2019.

Todo ello ha permitido publicar este mismo año la norma UNE 318002-3 “Técnicas de riego. Telecontrol de zonas regables. Parte 3: Interoperabilidad" y avanzar considerablemente en el desarrollo de la futura norma internacional ISO 21622-3 "Irrigation techniques - Remote monitoring and control for irrigation - Part 3: Interoperability".

\section{Materiales y métodos}

\subsection{Finca tecnológica del Centro Nacional de Tecnología de Regadíos (CENTER)}

El CENTER es un centro de referencia en el mundo del regadío y supone el escaparate perfecto de un proyecto interoperable que ya es una realidad. La finca seleccionada se compone de cuatro redes de riego diferentes, diferenciadas por sus necesidades de presión, caudal y por el sistema de riego que emplean. El total de hidrantes de riego, compuestos por válvula y contador, asciende a sesenta y siete unidades. Además, se cuenta con otros dos puntos de control desde los que se monitorizan sensores 


\section{Congreso Nacional de Riegos CARTAGENA 2021}

que recogen propiedades relacionadas con el suelo (conductividad, humedad y temperatura) y que forman también parte de la definición del estándar.

El total de entidades de riego a controlar supone un total de setenta y cinco. Previo al inicio de las pruebas, para su control, el CENTER disponía de treinta y cuatro unidades remotas del mismo fabricante (SIGA-TRAGSA), para controlar todas estas entidades de riego.

\subsection{Unidades remotas (sistemas) interoperables instaladas}

Para realizar las pruebas previstas, se han sustituido un total de doce de las unidades remotas existentes por equipos de otros sistemas interoperables, lo que supone un treinta y cinco por ciento del total. Las nuevas remotas controlan un total de diecisiete hidrantes de riego, un veinte siete por ciento del total. Las localizaciones donde se han instalado los nuevos sistemas pueden verse en la siguiente figura. Todas las entidades de riego que no aparecen señaladas siguen estando controladas por el sistema de telecontrol original que también se ha implementado para que fuese interoperable.

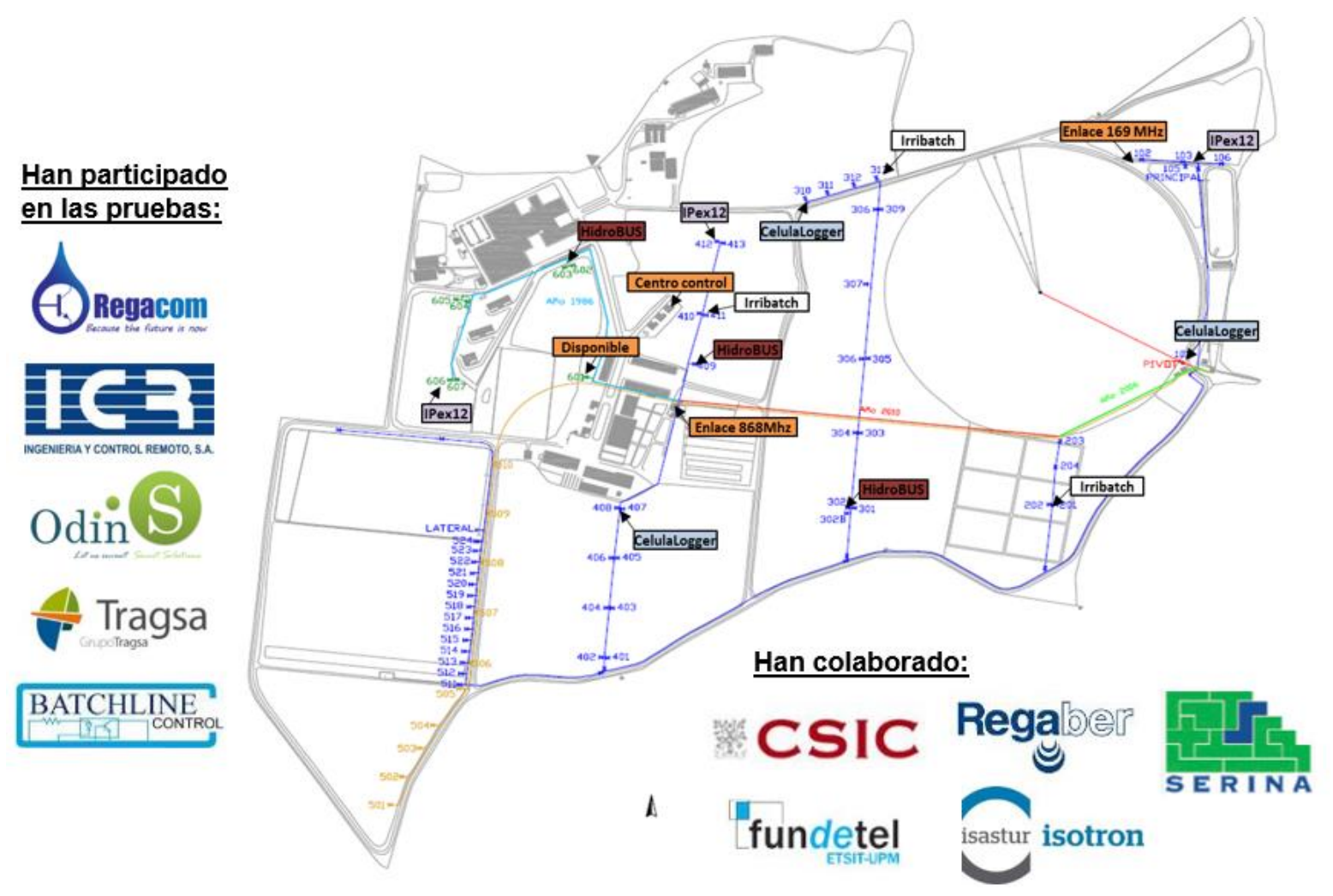

Figura 1. Despliegue de sistemas de telecontrol. 


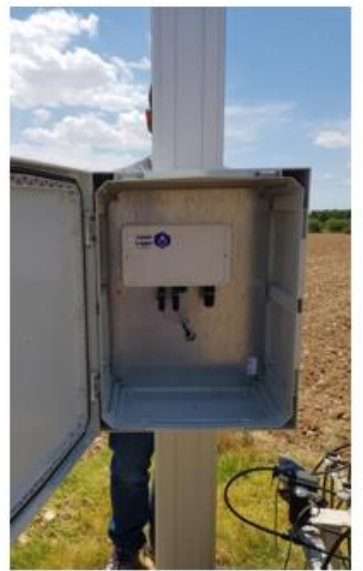

CelulaLogger

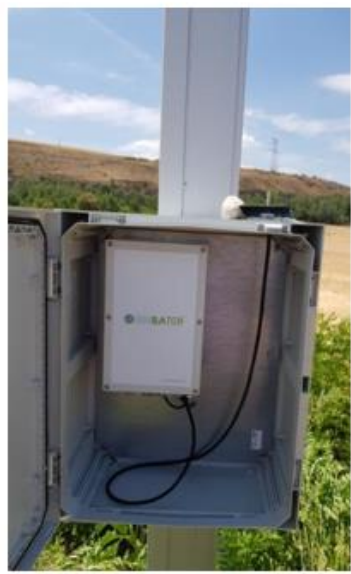

Irribatch

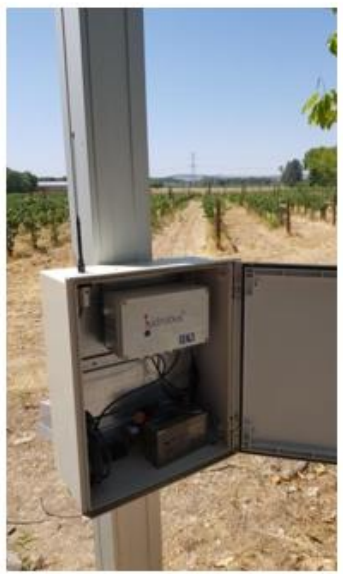

HidroBUS

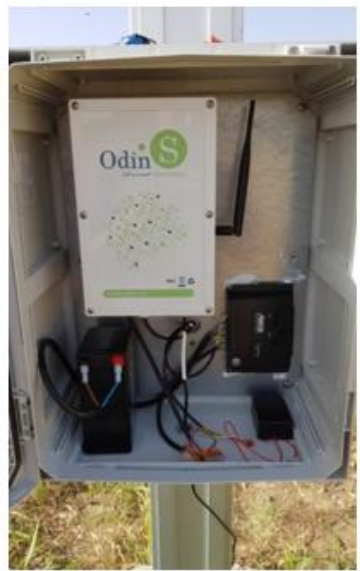

IPEx12

Figura 2. Sistemas interoperables, adicionales el existente del SIGA, instalados en el CENTER.

Tabla 1: Características de los sistemas interoperables instalados.

\begin{tabular}{l|c|c|c}
\hline \multicolumn{3}{c}{ Característcas Sistemas Interoperables } \\
\hline Sistemas & Comunicación & Arquitectura & Suministro de energía \\
\hline SIGA - Tragsa & RF 433 Mhz & Concentrador & Batería 12V 7 Ah+Panel solar 40W \\
\hline $\begin{array}{l}\text { CelulaLogger - } \\
\text { Regacom }\end{array}$ & GPRS & Directa & Pila litio o Panel solar 5W + Batería 12V \\
\hline Irribatch - Batchline & RF 869 Mhz & Concentrador & Batería 12 V 1 Ah + Panel solar 0,8 W \\
\hline Hidrobus - ICR & RF 869 Mhz & Concentrador & Batería 12V 7 Ah + Panel solar 5W o Pila \\
\hline IPEx12 - Odins & GPRS & Operador & Batería 12 V 7 Ah + Panel solar 5 W \\
\hline
\end{tabular}

Para poder ilustrar cómo beneficia la interoperabilidad en los diferentes sistemas instalados, se muestra en la tabla anterior que aún contando con diferentes tecnologías de comunicaciones (radio, GPRS), arquitecturas y sistemas de suministro de energía, no afectan a la interoperabilidad de los equipos 


\section{Congreso Nacional de Riegos CARTAGENA 2021}

\subsection{Herramientas para integración de datos y sistemas}

Para integrar los datos de todos los sistemas de telecontrol (subsistemas), se ha desplegado una herramienta de enrutamiento para realizar funciones de bróker/coordinador de mensajería en instalaciones de regadío y una herramienta de gestión para la recogida de datos e informes.

El estándar define como integrar datos de diferentes sistemas, con independencia de su origen y con mínimo impacto para el usuario. Para ello, se establecen las siguientes fronteras y niveles.

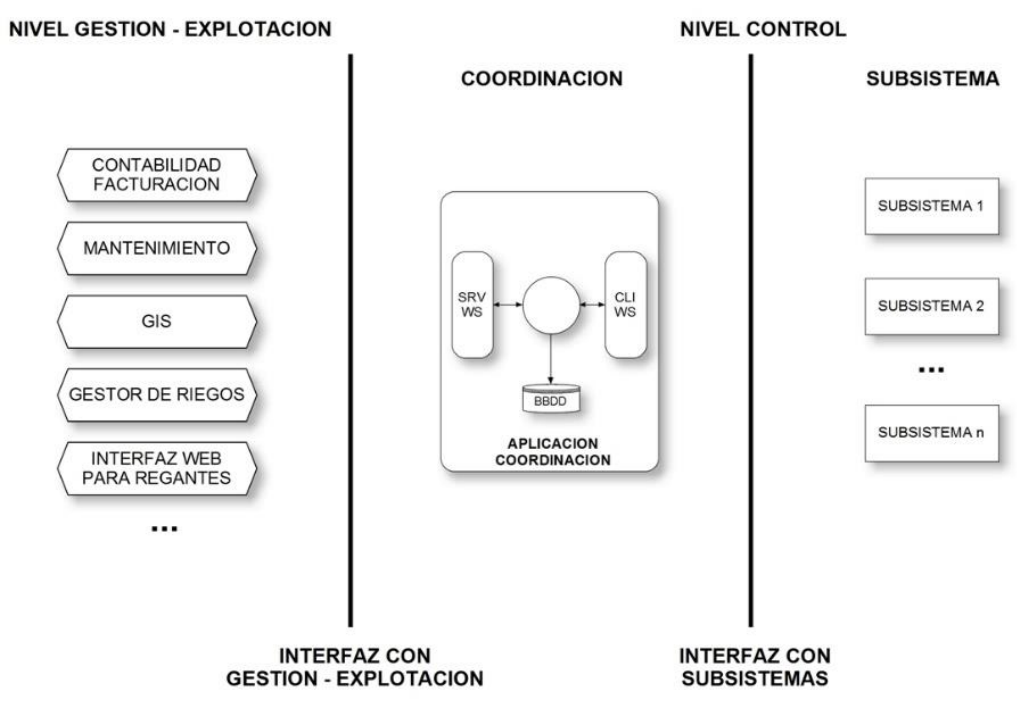

Figura 3. Arquitectura interoperable en el estándar.

\subsubsection{Bróker de coordinación}

Un bróker de mensajería es un mecanismo mediador de comunicación entre aplicaciones, que minimiza el grado de conocimiento mutuo entre aplicaciones para poder intercambiar mensajes, permitiendo su desacoplamiento. En el estándar de interoperabilidad esta herramienta recibe el nombre de bróker de coordinación.

¿Cómo funciona y cuáles son las funciones del bróker de coordinación?

El bróker de coordinación es un elemento software que proporciona interoperabilidad entre los sistemas de control y los sistemas de gestión, realizando las siguientes funciones básicas:

1. Mapeado de las entidades hidráulicas y asociación de éstas con los subsistemas que las controlan.

2. Enrutar las peticiones (lecturas, escrituras, programas de riego).

3. Transformar las peticiones al protocolo que soporte el destinatario. 


\section{Congreso Nacional de Riegos CARTAGENA 2021}

4. Recoger las respuestas y devolvérselas al emisor, transformándolas si es necesario.

5. Guardar información de las entidades de riego (identificación, localización, datos de diseño, datos para comunicar).

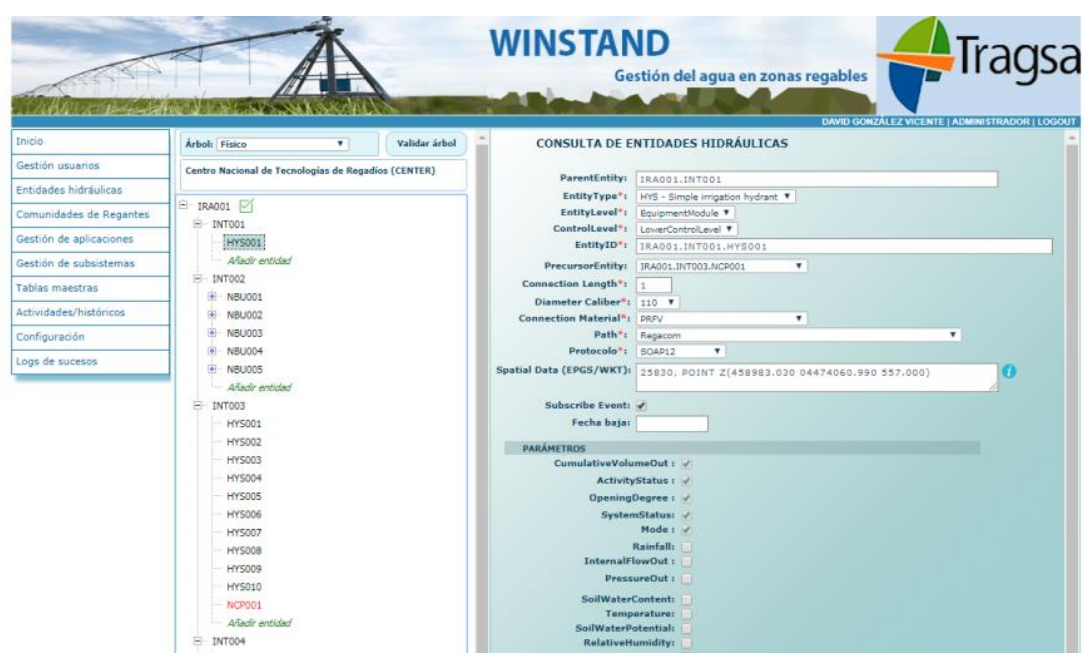

Figura 4. Interfaz de coordinación desarrollada.

En la norma se indica una definicion genérica no vinculada a ninguna tecnología concreta, pero para poder ponerlo en práctica, se han utilizado dos de las tecnologías actuales existentes como son SOAP y REST. REST es un lenguaje muy empleado en el mundo IoT. En el standard existe la documentación necesaria para que cualquier empresa interesada, pueda desarrollar su propio coordinador.

\subsection{Herramienta de gestión}

Para finalizar, el despliegue se completa con una herramienta de gestión utilizada a día de hoy en diferentes Comunidades de Regantes. Dicha herramienta se emplea para:

- Monitorizar el estado y recoger datos (valores de propiedades) de todos los hidrantes de la finca, así como de los puntos de control existentes.

- Enviar diferentes tipos de programa de riego.

- Monitorizar el resultado de los programas de riego ejecutados, revisando su duración, condición de finalización y consumo de agua asociado. 


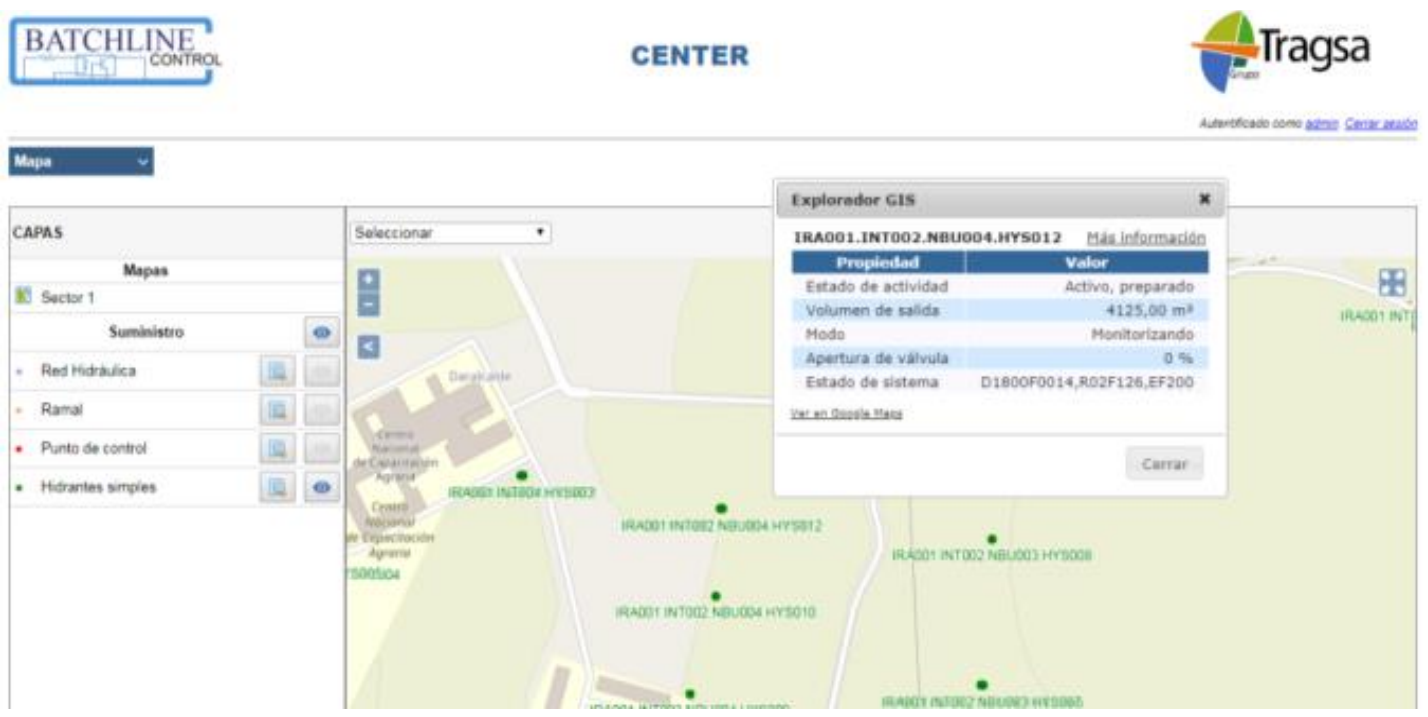

Figura 5. Aplicación de gestión implementada.

El desarrollo a nivel de software de esta aplicación lo realizó Batchline, pero al igual que sucede con el coordinador, cualquier empresa puede adaptar su producto a la norma interoperable.

\section{Resultados y discusión}

El calendario de pruebas se inició en junio de 2019, después la instalación de los nuevos equipos de control y todas las herramientas informáticos descritas. La primera campaña de riego finalizó a inicios del mes de noviembre. Para los dos años posteriores, se establecieron las mismas fechas como inicio y fin para el tiempo de estudio.

Dentro del primer año, se consideró la primera quincena como periodo de adaptación y ajuste de los despliegues. Los meses de noviembre no forman parte de la fase de estudio al ser un período poco representativo debido a la baja actividad de riego en ese tiempo. Así pues, los meses representativos para el estudio han sido los cuatro meses centrales de cada año teniendo en cuenta tres prioridades:

- Primera: responder a las necesidades de gestión del CENTER, cuyos aspectos centrales son el envío de programas de riego, monitorización y la recepción de informes de los datos obtenidos.

- Segunda: ensayar todas las posibilidades de intercambio en aquellos sistemas que lo soporten. Adicionalmente a las mencionadas en el punto anterior, el estándar incluye otras que son relevantes para la gestión del riego, destacando la sincronización de contadores, el paro o la cancelación de programas en ejecución. También se realizan pruebas relacionadas con las entidades de riego como son el alta y baja de entidades de riego.

- Tercera: desarrollar el mayor número posible de pruebas. Cubriendo las necesidades básicas reflejadas en las prioridades anteriores, se intenta realizar el mayor número posible de pruebas para asegurar que los resultados obtenidos son significativos. 


\section{Congreso Nacional de Riegos CARTAGENA 2021}

- Cuarta: realizar pruebas de fiabilidad en todos los componentes desplegados. Para ello, se emplean las peticiones masivas para el refresco de los datos, soportados por la herramienta de gestión. La prioridad es comprobar la estabilidad de los diferentes elementos al lanzarles una cantidad importante de peticiones.

Durante las tres campañas de riego se ha hecho un seguimiento de todas las pruebas realizadas, mostrándose a continuación, un resumen de los resultados generales del 2019 que son los más representativos al ser el primer año.

Tabla 2: Peticiones tramitadas.

\begin{tabular}{l|c|c}
\hline \multicolumn{3}{c}{ Peticiones tramitadas } \\
\hline Peticiones tramitadas & 2507 & \\
\hline Resultado 1: éxito & 1785 & $71,2 \%$ \\
\hline Resultado 2: éxito parcial & 574 & $22,9 \%$ \\
\hline Resultado 3: fallo & 148 & $5,9 \%$ \\
\hline
\end{tabular}

A efectos de interoperabilidad, tanto los resultados de tipo 1 como los de tipo 2 (éxito y éxito parcial), se consideran satisfactorios. Por ello, se puede concluir que la interoperabilidad ha funcionado correctamente en el 94,1\% durante el periodo de pruebas.

Tomando como referencia los cuatro meses de mayor actividad, principalmente el verano, el porcentaje medio de fallo en 2019 es del 5,9\% (148 errores sobre 2507 peticiones tramitadas), si bien se observa una tendencia a la baja en el porcentaje de errores registrados en los siguientes años reduciendose a un 5,5\% en el 2020 y a un 5,3\% en el 2021 Ha de tenerse en cuenta que dentro de los errores incluidos en el resultado 3 , se incluyen sólo aquellos que no han sido generados por pruebas erróneas y que son debidos a:

- Fallos de comunicaciones: cuando las peticiones no se tramitan correctamente;

- Fallo específico de comunicaciones por timeout: cuando las peticiones realizadas no reciben respuesta en el tiempo estipulado por el emisor, asimilándose a un error de interfaz interoperable;

- Problemas de seguridad: cuando uno de los componentes interoperables bloquea la tramitación de peticiones debido a criterios de seguridad informática (autenticación, direccionamiento, etcétera), asimilándose a un error de interfaz interoperable;

- Errores de implementación: cuando la interfaz interoperable ha funcionado correctamente pero el destinatario no interpreta correctamente una petición bien formulada.

La distribución concreta que se ha producido por tipos de error se muestra en la tabla siguiente: 


\section{Congreso Nacional de Riegos CARTAGENA 2021}

Tabla 3: Clasificación de errores.

\begin{tabular}{l|c|c}
\hline \multicolumn{3}{c}{ Clasificación de errores } \\
\hline Total de errores & 148 & \\
\hline Fallo de comunicación y Timeout & 33 & $22,3 \%$ \\
\hline Problema de seguridad & 34 & $23,0 \%$ \\
\hline Errores de implementación & 81 & $54,7 \%$ \\
\hline
\end{tabular}

\section{Conclusiones}

Tras tres campañas de riego utilizando herramientas y sistemas interoperables que han hecho posible la monitorización de los parámetros básicos del riego, como son las presiones y volúmenes suministrados, la ejecución, con éxito, de numerosos programas de riego, la recogida de sus informes, la sincronización de contadores y la recogida de datos históricos, entre otras funcionalidades, se pueden sacar las siguientes conclusiones:

1- En una red de riego real se puede implementar un sistema de telecontrol totalmente interoperable, no sólo en instalaciones nuevas sino también en sistemas que ya están en funcionamiento.

2- La interoperabilidad favorece el desligamiento entre usuarios y fabricantes de sistemas de telecontrol, permitiendo la elección de la mejor solución, fomentando la competitividad y mejora de los productos ofertados, además de la apertura del mercado de prestación de servicios de explotación y mantenimiento, reducción del abandono de los sistemas y ptenciación del uso de las informaciones que de ellos se puede extraer.

3- Los sistemas interoperables facilitan el manejo y la gestión al usuario ya que podrá adquirir datos de diferentes sistemas de control en una única herramienta de gestión, favoreciendo la toma de decisiones y el control de su instalación.

4- Los fabricantes de telecontrol también se verán beneficiados al poder incluir una funcionalidad que ofrece muchas posibilidad a los usuarios.

5- Tanto la norma UNE 318002-3 “Técnicas de riego. Telecontrol de zonas regables. Parte 3: Interoperabilidad" ya publicada, como la futura norma internacional ISO 21622-3 "Irrigation techniques - Remote monitoring and control for irrigation - Part 3: Interoperability" establecen los requisitos mínimos que debe cumplir un sistema para ser interoperable pero no obligan a los fabricantes a modificar su hardware o su software de control. Únicamente se solicita una capa intermedia que sea capaz de traducir sus mensajes para que sean entendibles por la aplicación de coordinación.

Acabada la fase de desarrollo y pruebas y una vez publicada la norma, se emplaza a los fabricantes de sistemas de telecontrol y a los desarrolladores de herramientas de gestión a implementar el estándar en sus productos. Del mismo modo, se emplaza también a los promotores y proyectistas a tener en cuenta criterios de interoperabilidad en la fase de diseño y desarrollo de proyectos relacionados con el control y la gestión de instalaciones de regadío. 